\title{
Computational efficiency for the surface renewal method
}

\author{
Jason Kelley and Chad Higgins \\ Dept. of Biological and Ecological Engineering, Oregon State University, 116 Gilmore Hall, Corvallis, OR 97333, USA
}

Correspondence: Jason Kelley (kelleyja@oregonstate.edu)

Received: 20 April 2017 - Discussion started: 1 June 2017

Revised: 5 March 2018 - Accepted: 15 March 2018 - Published: 16 April 2018

\begin{abstract}
Measuring surface fluxes using the surface renewal (SR) method requires programmatic algorithms for tabulation, algebraic calculation, and data quality control. A number of different methods have been published describing automated calibration of SR parameters. Because the SR method utilizes high-frequency $(10 \mathrm{~Hz}+)$ measurements, some steps in the flux calculation are computationally expensive, especially when automating SR to perform many iterations of these calculations. Several new algorithms were written that perform the required calculations more efficiently and rapidly, and that tested for sensitivity to length of flux averaging period, ability to measure over a large range of lag timescales, and overall computational efficiency. These algorithms utilize signal processing techniques and algebraic simplifications that demonstrate simple modifications that dramatically improve computational efficiency. The results here complement efforts by other authors to standardize a robust and accurate computational SR method. Increased speed of computation time grants flexibility to implementing the SR method, opening new avenues for SR to be used in research, for applied monitoring, and in novel field deployments.
\end{abstract}

\section{Introduction}

Originally described by Van Atta (1977), the SR model measures vertical flux that occurs during rapid events which manifest as coherent structures in a turbulent flow. The physical mechanisms are statistically distinct from those described in the eddy covariance (EC) method, which has been established as a robust and accurate method to measure flux (Baldocchi, 2014). The surface renewal (SR) method offers several advantages and complements the use of EC to measure flux. While EC requires fast $(10 \mathrm{~Hz}+)$ measurement of both the vertical wind speed and air temperature to measure the sensible heat flux, the SR method does not explicitly require vertical wind speed, allowing flux to be determined solely from rapid measurements of temperature or other scalar concentrations. Because fewer, lower-cost sensors are required, the SR method theoretically can be used for general applied monitoring (Paw U et al., 2005; Spano et al., 2000). Another advantage of SR is the ability to measure flux very near the surface or near the top of the plant canopy (Katul et al., 1996; Paw $U$ et al., 1992). By taking measurements very close to the surface, the measurement fetch is reduced and the effective "flux footprint" is smaller (Castellví, 2012), yielding a more localized flux estimate.

The SR method estimates turbulent transport rates from fast response measurements of scalar properties such as temperature or trace gas concentration. In the SR conceptual model, rapid changes in scalar concentration are associated with episodic displacement of near-surface air parcels, and the surface condition is renewed from upper air. While in proximity to the surface, the air parcels are gradually enriched or depleted in temperature or scalar concentration by diffusion (Castellví et al., 2002; Paw U et al., 1995). The majority of flux from the surface is attributed to these rapid ejections, which distinguish coherent structures in near-surface atmospheric motions (Gao et al., 1989). The duration and amplitude of these rapid fluctuations (visible as ramps in the scalar trace) are used to determine the magnitude and direction of the flux density. Because of the short duration of these events, the SR method complements spectral methods to evaluate the flux contributions made over timescales shorter than the typical 15-30 min averaging time used for EC (Katul et al., 2006; Shapland et al., 2012a, b). Rapid flux measurement will facilitate new applications, such as spatial mapping of flux using vehicle-mounted, near-surface sensors, and real-time monitoring systems. Mobile SR im- 
plementations and other novel field methods could provide new insights into the complexities of sub-basin-scale hydrology, be used to validate downscaled models, and measure the heterogeneity of flux at sub-field scales.

The implementation of SR requires a prescribed averaging time period (on the order of minutes) and ramp time duration (on the order of seconds), for which a representative and statistically robust flux magnitude can be determined. To implement SR on a moving vehicle (for instance, to map spatially variable flux), finding a minimum averaging time is desirable to increase the spatial resolution of the resulting map. The averaging time and lag time used in the SR method relate the sensitivity of the scalar measurement to the timescales at which most significant flux occurs (Shapland et al., 2014). To find the minimum measurement period, field studies were conducted in 2014 and 2015 over various types of surface conditions. This required a rapid computational method that worked over a range of different time averaging periods, and which could implement the various calibration procedures used in the SR method. Initial attempts to calculate flux followed methods as described by Paw U et al. (2005) and Snyder et al. (2008). However, implementing these methods as documented was hampered by slow computation time, which constrained the many iterations required to determine the minimum flux averaging period.

Open-source software and online forums are abound with methods that utilize advances in computing power, memory availability, and the accessibility of multithreaded processing. These methods reduce computational overhead, and can augment the SR technique to allow implementation with lowcost computers and data loggers, or where remote telemetry is required. Three example methods are shown here which streamline specific operational steps in the SR method. The first is a method adapted from signal processing to "despike" noisy data, a quality control technique commonly used in processing raw meteorological data. Second is a method to compute structure functions over multiple time lags rapidly using convolution in two dimensions. Third, an algebraic array calculation is used to find the cubic polynomials roots used to determine the SR ramp amplitude. By using more efficient algorithms, rapid iterative trials can be conducted to adjust calibration parameters, test hypotheses on the time averaging of flux calculations, and potentially measure SR flux in real time.

Advantages such as low-cost, relatively simple instrumentation, and easier field implementation are all cited as motivating factors to use the SR method (Paw U et al., 2005), yet work remains to standardize a robust method (French et al., 2012; Suvočarev et al., 2014). Because sensor cost is reduced, SR systems can be implemented to measure flux more extensively than EC, and in situations where EC is impractical. Extensive, site-specific SR estimates can augment the utility of sparsely located, permanent weather stations in mapping the heterogeneity of surface flux. Examples of situations which could benefit from low-cost flux mea- surements include direct crop ET monitoring, experiments at remote field sites, and developing regions. While SR may expand flux measurement applications, the method still requires standardized calibration and quality control measures to establish that SR is robust and accurate, and a critical step in developing the method is to reduce computation costs.

\section{Methods}

The example algorithms shown here improve or economize existing calculation methods, including despiking of time series data (Højstrup, 1993; Starkenburg et al., 2016), calculation of structure functions (Antonia and Van Atta, 1978), and Fourier analysis of signals, i.e., spectral analysis (Press, 2007; Stull, 1988). In each case, dramatically faster execution times were accomplished using simple programming improvements. Most efficiency gains were a result of code vectorization, which is the conversion of iterative looping algorithms into array calculations. All methods described here were implemented in the MATLAB language (The Mathworks Inc., 2016), with the Statistics, Curve Fitting, and Signal Analysis toolboxes. MATLAB's Profiler (profile.m) was used to track the memory demand and time to implement calculations. Trials were conducted on multiple desktop systems; for uniformity, analysis shown here only used test runs that were conducted on a Windows 10 operating system running on an Intel Core ${ }^{\mathrm{TM}}$ i7-3720QM processor operating at $2.60 \mathrm{GHz}$ with $16 \mathrm{~GB}$ of RAM. Processor clock speed was verified using MATLAB's Profiler tool at run time, and processing times reported are described as run time (actual observed execution time) or as total run time, which is the sum of CPU time for all calculation threads. Example methods are indicated by function name in italics. Abbreviated, commented scripts for the example functions are provided in the Supplement. Example data were collected during various field experiments from 2014-2017, using an integrated sonic anemometer and infrared gas analyzer (IRGASON) and fine wire thermocouples (FWTs), and were recorded at 10,20, and $100 \mathrm{~Hz}$ using a CR1000 data logger (Campbell Scientific). The data used in verifying the methods are provided through supplementary materials online (https://doi.org/10.7267/N9X34VDS).

Vector and array calculations are more efficiently executed than iterative methods; vectorization of MATLAB code entails removing loops (which are not pre-compiled) and taking advantage of implicit parallel methods in MATLAB's precompiled functions (Altman, 2015). Other significant improvements were enabled through the fast Fourier transform by using convolution of number arrays, rather than iterative operations. In the case of determining ramp geometry in the SR method, Cardano's solution for depressed cubic polynomials (published in 1545) reduces a root-finding algorithm from an iterative numerical approximation to an exact algebraic vector calculation. While some of the implementation 


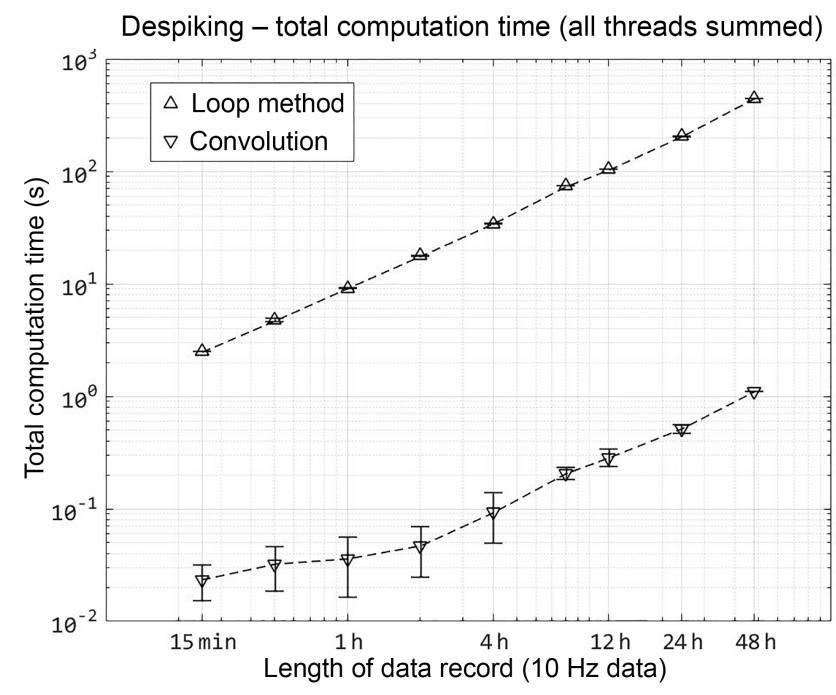

Figure 1. The total computation time is the sum of CPU time spent on all calculation threads. Triangles mark the mean run time for multiple runs, which varied from 30 runs ( $15 \mathrm{~min}-4 \mathrm{~h}$ data) to 10 runs $(8,12,24 \mathrm{~h})$. The $48 \mathrm{~h}$ calculation is represented by one run only. Error bars represent 1 standard deviation of all runs.

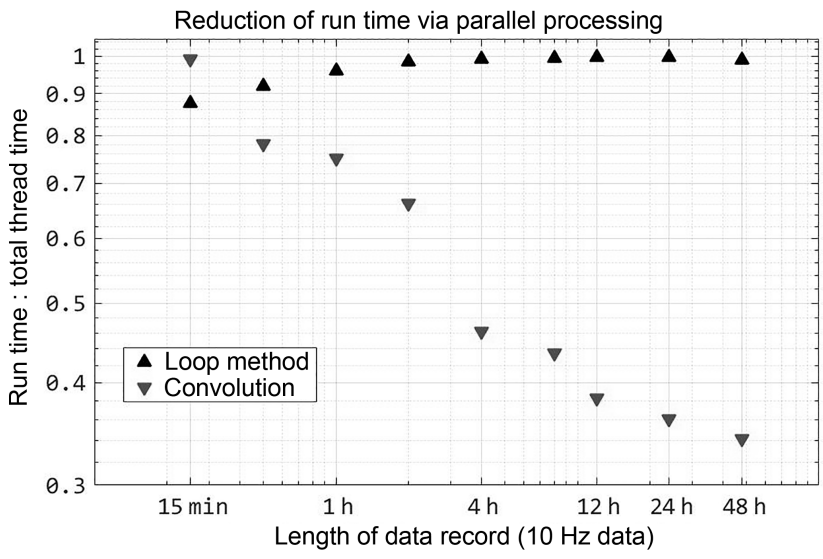

Figure 2. Fast calculation of larger data sets is due to implicit parallel processing via the FFT, which is readily performed by multiple simultaneous threads. The efficiency of parallel processing is shown by a lower ratio of run time to total thread time.

of these methods are particular to the MATLAB language, the general mathematical concepts are universal. Although these methods were prototyped in MATLAB, the examples shown are generally useful as solutions to challenges commonly encountered in micrometeorology.

\subsection{Despiking of noisy data using convolution}

Despiking is the removal of erroneous or extreme data points from a time series of sampled values. It is a common procedure when measuring environmental parameters, especially in challenging conditions or complex environments
(Göckede et al., 2004; Starkenburg et al., 2016). The origin of spikes in a time series may be electronic or physical (sensor malfunction or actual physical non-errors); regardless of the origin, spikes can be recorded as abnormally large or small values, or may be marked by an error flag defined in the firmware. Spikes become problematic if they are not readily differentiated during automatic data imports (Rebmann et al., 2012). Spikes interfere with statistical calculations, and require some deliberate and objective method to identify, remove, and interpolate where they exist. For instance, a data logger program may record an error as "9999" or a character string, while MATLAB denotes missing values in a numerical array as NaN ("not a number"). Because normally distributed data may contain noise in a wide range of values, robustness of the despiking algorithm is complicated by the requirement to differentiate between "hard spikes" characteristic of automatic flags (such as 9999) and "soft spikes", which are realistically valued but erroneous measurement. An objective limit for soft spikes is usually defined as appropriate for the signal-to-noise ratio of any particular data, usually in terms of variance during a defined windowing period. Clearly distinguishing errors can be achieved by a static objective criteria, by a dynamic statistic, or in a separate preprocessing operation. Previous authors have described a variety of methods including use of autocorrelation (Højstrup, 1993) and statistics within a moving window (Vickers and Mahrt, 1997). A comprehensive review of despiking methods is presented by Starkenburg et al. (2016), with emphasis on the accuracy and statistical robustness of different computation methods.

Despiking is a problem of conditional low-pass filtering; consequently this procedure can be treated as an application of signal processing which can be performed efficiently using convolution. Mathematically, convolution can be understood as a multiplicative function that combines a data signal with a filter signal. For a discrete signal, the filter is a weight array which is multiplied (in the Fourier domain) with data inside a window. In the time domain, the window can be visualized as moving along the data array as it is multiplied. As examples, a filter with a weight of 2 at the center of the window, and zeros elsewhere, would amplify the data signal by a factor of 2; a filter 10 samples wide, each weighted at 0.1 , generates a running average of the data. The computational efficiency of convolution is a product of the fast Fourier transform (FFT), which allocates memory efficiently by a process known as bit switching. A thorough treatment of bit switching can be found in Chapters 12 and 13 of Press (2007). To demonstrate the increased efficiency of the FFT, two methods were used to despike $8.5 \mathrm{~h}$ of $20 \mathrm{~Hz}$ sonic temperature data (609 139 samples). One method utilized a for loop, following the objective criteria described by Vickers and Mahrt (1997). The second method (shown in despike.m) used convolution to determine a running mean and standard deviation used in the identification of spikes. After multiple runs with different input criteria, the first pro- 
gram average run time was $27 \mathrm{~s}$. Using convolution, the second program average run time was $0.2 \mathrm{~s}$, decreasing run time by approximately $99 \%$. While this drastic improvement may potentially overemphasize slow compile times of for loops in MATLAB (compared to other languages), it nonetheless demonstrates the value of the FFT in calculations with time domain signals. Faster processing time facilitates more comprehensive, calibrated, and accurate analysis, and can reduce data loss compared to coarser filtering techniques.

To test computation time uniformly, identical $10 \mathrm{~Hz}$ data were sub-sampled to record lengths of 0.25 to $48 \mathrm{~h}$, and multiple runs were despiked with each sample set. Raw data were checked for hard error flags which required converting text to number values. Data were not otherwise manipulated prior to despiking. MATLAB Profiler was used to track the run time for all threads, using the undocumented flag "built-in" to track pre-compiled MATLAB functions as well as user functions, ${ }^{1}$. The total run time for all threads was tabulated and averaged across sets of each data length (Fig. 1). By using convolution, despiking was 2 orders of magnitude faster for all lengths of data. To illustrate the effect of MATLAB's built-in parallel processes, Fig. 2 shows the ratio of actual run time to total run time, indicating that the convolution method relies on computations conducted in parallel for processing increasingly longer data records. This benefit is directly accrued from the efficiency of the FFT.

With increased computation speed, automatic and accurate despiking can be accomplished, with reduced time cost to determine any necessary calibrate for the procedure. The various methods employed to despike data are variously limited by computational inefficiency (Starkenburg et al., 2016). "Phase space thresholding", originally described by Goring and Nikora (2002), is one such method that Starkenburg noted as being hampered by computational costs, and by a requirement for iterative applications to calibrate despiking parameters. By decreasing the execution time, a similar method was developed that allows rapid and accurate despiking of data, for the detection of both hard and soft spikes. A phase space method allows objective criteria to be calibrated for specific sensor data, and a visual diagnostic phase space diagram that allows for rapid calibration of the despiking criteria (Fig. 3). Projecting the signal into a phase space diagram reveals modes related to sensor error, response time, and other factors leading to spikes. Using convolution to determine moving window statistics (such as a moving mean, standard deviation), objective identification of behaviors characteristic to a particular sensor response. In Fig. 3, infrared gas analyzer data (in this case, signal strength) collected at $20 \mathrm{~Hz}$ for 17 days are projected with 1 min moving window statistics. Based on this projection, a cut-off in phase space for spike identification can be assigned, and the subsequent percentage of removed data calculated. In this case, the sensor was

\footnotetext{
${ }^{1}$ http://undocumentedmatlab.com/blog/ undocumented-profiler-options-part-4 last access: January 2017
}

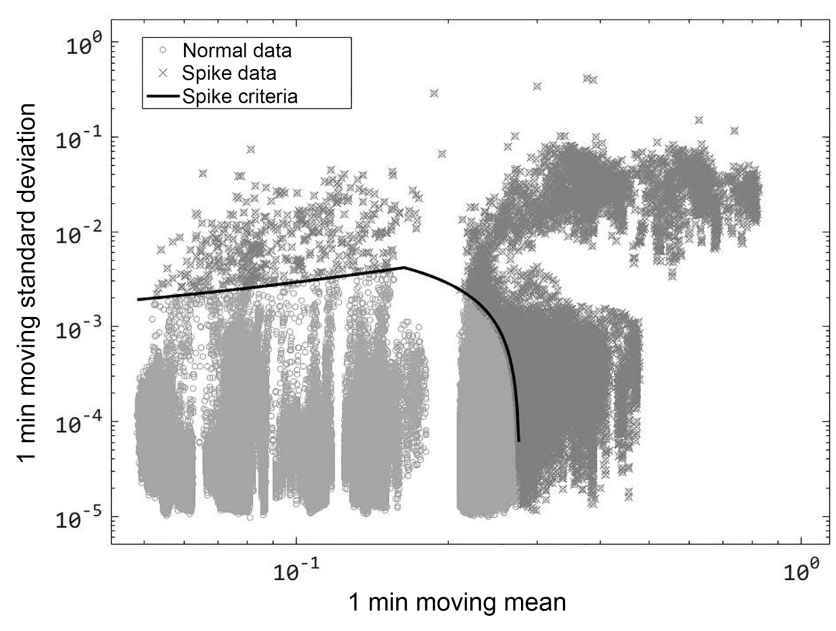

Figure 3. Phase space diagram showing moving window statistics of IRGA signal (17 days of $20 \mathrm{~Hz}$ data).

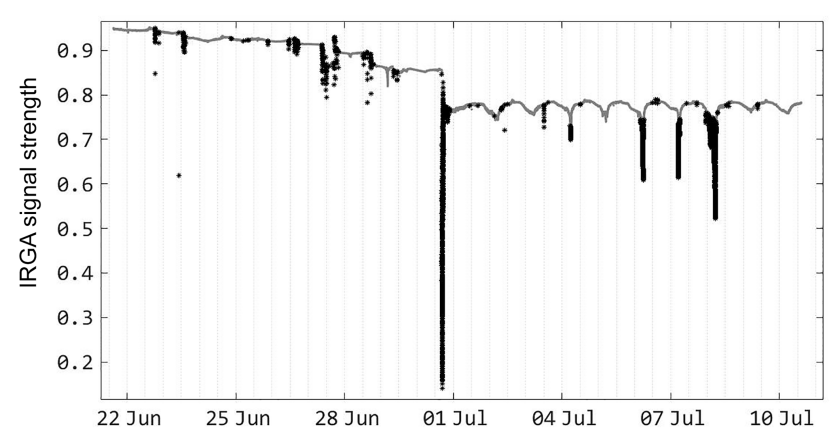

Figure 4. Time series showing data removed as spikes (bolded) from phase space criteria in Fig. 3.

repeatedly affected by dust from farm operations (Fig. 4), yet only $1.5 \%$ of the data were required to be removed as spikes due to the precision of the despiking algorithm. This procedure took less than $5 \mathrm{~s}$ of computation.

\subsection{Structure function calculation}

Another computationally intensive process in SR is the determination of the second-, third-, and fifth-order structure functions. Ramps are an identifiable feature in the measured temperature trace above any natural surface, yet determining the characteristic ramp geometry from high-frequency data requires an efficient, robust, and preferably automated procedure. There are several methods to determine ramp geometry, including visual detection (Shaw and Gao, 1989), lowpass filtering (Katul et al., 1996; Paw U et al., 1995), wavelet analysis (Gao and Li, 1993), and structure functions (Spano et al., 1997). Structure functions in particular provide both objective criteria to detect ramps and an efficient method to tabulate statistics of time series data, and use of structure functions has become the predominant method used for SR. 
The general form for a structure function is

$S^{n}(r)=\frac{1}{N-r} \sum_{1}^{i=N-r}[T(i+r)-T(i)]^{n}$,

in which a vector of length $N-1$ is composed of differences between sequential (temperature) samples $T(i)$, separated by lag $r$. The structure function $S^{n}(r)$ of order $n$ for a given sample lag $r$ is obtained by raising the difference vector to the $n$ power, summing the vector and normalizing by $N-1$. In a turbulent flow field, the sampled fluctuations of scalar time series $\Delta T(i)$ are a combination of random fluctuations and coherent structures (Van Atta and Park, 1972). The random (incoherent) part of the signal is a product of isotropic turbulent processes, and over an adequately large sample this sample should have no particular directional sense or orientation (by the isotropic definition). On the other hand, coherent structures generate characteristic anisotropic signatures, with periods of gradually change punctuated by sharp transitions. These sharp transitions occur during "sweeps and ejections" of parcels enriched or depleted in scalar concentration (heat or water trace gas), evidence of transport from an Eulerian perspective. Structure functions can be used to decompose the time series fluctuations into isotropic and anisotropic components and identify the characteristic ramp amplitude and duration of coherent structures (Van Atta, 1977). Advances in sensor response time and processor speed have revealed an increasingly detailed picture of the coherent ramp structures. In deriving a method to find ramp geometry, Van Atta (1977) calculated structure functions for eight different lags. Two decades later, increased processor power and memory size allowed Snyder et al. (1996) to calculate structure functions on $8 \mathrm{~Hz}$ data for lags from 0.25 to $1.0 \mathrm{~s}$, but they were unable to resolve fluxes accurately at some measurement heights and surface roughness conditions. Later it was realized that determining the contributions from "imperfect ramp geometry" would require more thorough examination of ramp durations (Chen et al., 1997a; Paw U et al., 2005).

For this analysis, data were used from several field experiments. The data records used ranged in length from $8 \mathrm{~h}$ to over 2 months, with sampling frequencies of 10,20 , and $100 \mathrm{~Hz}$ (fastest frequency for short duration trials only). Initially, computation of structure functions with the first method (series of nested for loops) for 3 min periods with lags up to $10 \mathrm{~s}$ required an average $39 \mathrm{~s}$ computation time. In contrast, using the convolution method, this same calculation was accomplished in $7.6 \mathrm{~s}$, an $\sim 80 \%$ reduction in execution time. The function strfnc.m (provided in Supplement, Sect. S1) also simultaneously time stamps the averaging period, finds the sign of $S^{3}(r)$ (used to find flux direction), and indexes the maximized value of $S^{3}(r) / r$, preparing the data for subsequent steps in determining flux. Using $100 \mathrm{~Hz}$ FWT data increased processing time using the convolution method to $38.4 \mathrm{~s}$. The loop method would be unable to process $100 \mathrm{~Hz}$ data in real-time applications, and would re-

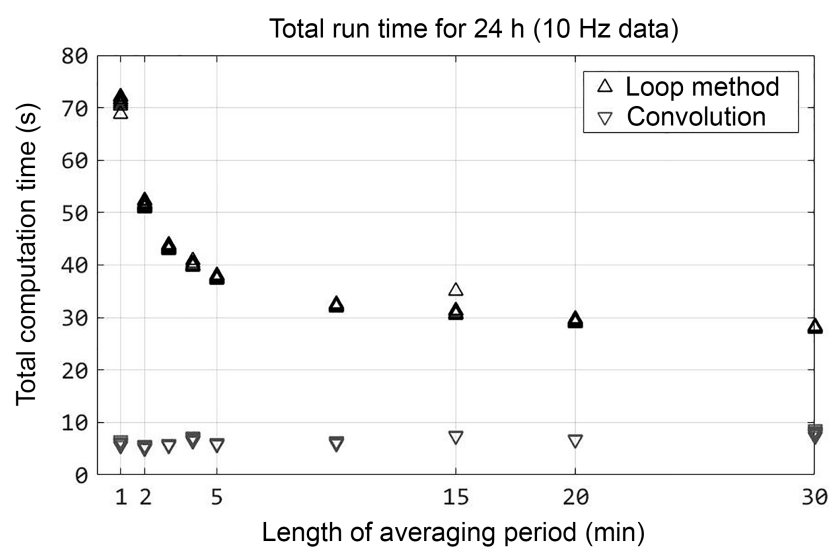

Figure 5. Ten iterations of the structure function calculations using a range of averaging periods.

quire long calculation time when using large continuous data records.

For a total of $N$ sample lags, two-dimensional convolution is performed using a filter matrix which is composed of $N$ column vectors of length $N+1$ : [1 - 1000], [10 - 100], [1000. - 1]. Each column represents a sample lag increasing distance. When the filter matrix is convolved with time series data, the column vectors of the resulting matrix are vectors of the element-wise differences $(T(i+r)-T(i))$ as in Eq. (1); these vectors correspond to each sample lag in the filter. Trials of $10 \mathrm{~Hz}$ data using MATLAB's Profiler showed that calculation efficiency is not accrued directly from convolution, but by changing the order of implementation. In the looping method, exponentiation $(n=2,3,5)$ is conducted on the difference vectors for each lag separately. The accelerated exponentiation in strfnc.m is possible by using matrix multiplication on the convolved matrix, and is faster due to compact memory allocation of the FFT. The resulting efficiency (calculation time for a given data size) does not depend on total data size, but is strongly dependent on the length of the averaging period used to partition the data (Fig. 5). In other words, the choice of averaging period length is the most significant factor in computation time of the maximized structure functions used to determine ramp geometry. Computation time increases rapidly for periods shorter than $5 \mathrm{~min}$. The length of averaging time is a critical consideration in developing a rapid SR measurement method.

In most SR studies to determine flux, a lag time is assigned to the structure function calculation, with only a few authors allowing for a procedure to maximize the ratio $S^{3}(r) / r$ (Shapland et al., 2014). Yet lag time has been identified as a critical parameter in the linear calibration of ramp geometry to calculate flux (French et al., 2012).

Because the ideal SR calculation identifies the lag which maximizes the ratio $S^{3}(r) / r$, the strfnc. $m$ procedure calculates structure functions for a continuous range of lags up to an assigned maximum lag. Based on repeated trials over a 


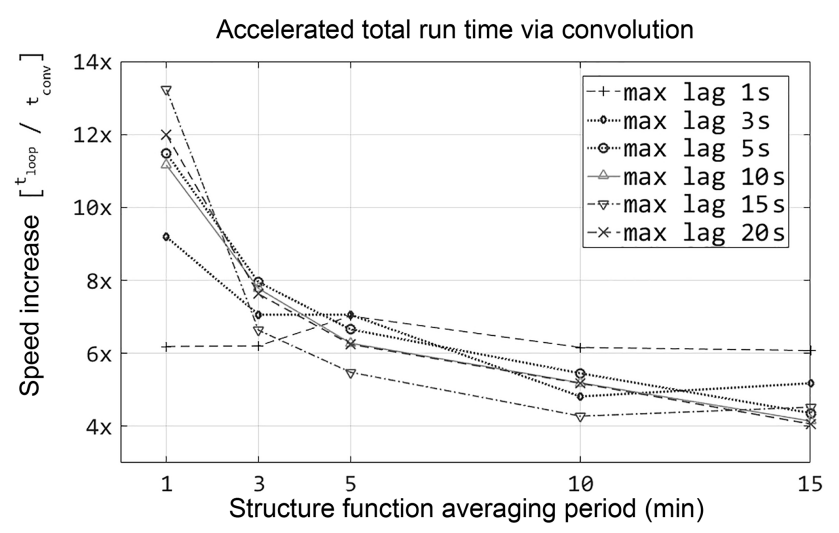

Figure 6. The performance gains using convolution are more significant for short averaging periods, regardless of maximum lag used in calculating structure functions.

broad range of stability conditions, a short maximum lag (3$5 \mathrm{~s}$ ) is usually adequate under unstable conditions. Following the model of parcel residence time, this is likely a result of buoyancy and higher flux magnitude leading to shorter ramp duration. Under stable conditions, though, longer lags are required to detect the true maximum of the ratio $S^{3}(r) / r$, indicating that the timescale contributing to flux increases. To evaluate sensitivity to the maximum calculated lag, the structure functions were calculated iteratively, varying the averaging period and maximum tested lag time (Fig. 6). Regardless of the length of the assigned range of lags, the convolution method was between 4 and $14 \times$ faster than the loop method, with short averaging periods again the largest factor in the difference between the two methods. Using the 2-D convolution, automated selection of a lag in a continuous time series is feasible.

\subsection{Cardano's method for depressed cubic polynomials}

For the idealized SR method, the structure functions are retained (for each averaging period) for the lag which maximizes the ratio $S^{3}(r) / r$ - this lag is associated with the theoretical maximum contributing scale of flux. The resulting values are used as coefficients in a cubic polynomial, the root of which is the ramp amplitude $(A)$ used to calculate flux:

$A^{3}+\left(10 S^{2}(r)-\frac{S^{5}(r)}{S^{3}(r)}\right) A+10 S^{3}(r)=0$.

The magnitude of the real root (of 3 possible roots) is the characteristic ramp amplitude of the scalar trace (Spano et al., 1997). The MATLAB root-finding algorithm computes eigenvalues of a companion matrix to approximate the solution to a $n$ th-order polynomial, regarding the input function as a vector with $n+1$ elements (roots.m documentation ${ }^{2}$ ).

\footnotetext{
${ }^{2}$ http://www.mathworks.com/help/matlab/ref/roots.html, last access: 9 August 2016
}

Consequently, this function cannot be executed directly on an array. On the other hand, an algebraic solution method can be applied to vectors. An appropriate method for this type of cubic polynomial was found by Gerolamo Cardano in the 1545 Ars Magna. Cardano's solution for "depressed" cubics (with no squared term) is found by substituting A with $\left(m^{1 / 3}+n^{1 / 3}\right)$ into the abbreviated equation $A^{3}+p A+q=0$. Expanding terms and using the quadratic equation yields an exact solution:

$A=\left(-\frac{q}{2}+\sqrt{\left(\frac{q}{2}\right)^{2}+\left(\frac{p}{3}\right)^{3}}\right)^{\frac{1}{3}}+\left(-\frac{q}{2}-\sqrt{\left(\frac{q}{2}\right)^{2}+\left(\frac{p}{3}\right)^{3}}\right)^{\frac{1}{3}}$,

where $p$ and $q$ are coefficients in the depressed cubic and derived from the structure functions (Edwards and Beaver, 2015). The function cardanos.m was adapted from a function by Bruno Luong, ${ }^{3}$, in a reduced form for the real-valued cases used to implement the SR method. The function output was verified against the MATLAB function roots. $m$ for polynomials with both positive and negative real valued inputs (imaginary inputs are applicable to ramp parameters). Solution for the real roots in this manner expedites determination of flux magnitude and direction. The algebraic root-finding method simplifies and speeds iterative application of the SR method by operating directly on arrays.

Solving for the roots of this function yields a single, predominant ramp amplitude from a given temperature trace (with units of ${ }^{\circ} \mathrm{C}$ or $\mathrm{K}$ ). In addition to ramp amplitude, the timescale or ramp duration must also be determined. Van Atta (1977) suggested that ramp time $\tau$ should be related linearly to amplitude $A$ and proposed

$\tau=\frac{-A^{3} r}{S^{3}(r)}$

In practice, determination of ramp time $\tau$ from $A$ using this equation requires an empirical calibration; this calibration has been shown to be related to surface conditions and instrumentation (Chen et al., 1997b; Shapland et al., 2014). Ongoing work using replicate measurements at multiple heights (Castellvi, 2004) and frequency response calibration (Shapland et al., 2014; Suvočarev et al., 2014) has begun to resolve the causes of variability in this parameter. In this study, it was found that the ratio in Eq. (4) remains essentially constant for a given surface roughness condition, allowing determination of $\tau$ algebraically. Automated computation of Eq. (2) using the exact solution facilitates rapid evaluation of the ramp geometry, and determining flux magnitude from ramp geometry is a relatively simple matter of linear scaling when calibrating to a control measure such as eddy covariance.

\footnotetext{
${ }^{3}$ https://de.mathworks.com/matlabcentral/fileexchange/ 27680-multiple-eigen-values-for-2x2-and-3x3-matrices access: 9 April 2018
}

last 


\section{Conclusions}

As with other methods for measuring flux from the surface, analytic solutions do not always translate easily into straightforward numerical computation, especially when working with large data records or when calculating in real time. In applied research, custom algorithms are often developed by individual researchers, requiring special training in programming, significant time investment, and the motivation to use sophisticated techniques that fully utilize available memory and processing power. Efforts to standardize the eddy covariance method (Aubinet et al., 2012; Baldocchi, 2014) and data quality control (Allen et al., 2011; Foken et al., 2012) have not yet been similarly applied to the SR method, although substantial work has been made to validate and unify SR methods (Castellví, 2012; Chen et al., 1997b; French et al., 2012; Suvočarev et al., 2014). By appropriating methods common in signal processing, and by sharing open-source tools on online forums, more sophisticated approaches can be implemented. In particular, reducing the computational overhead of calculating flux enables broad implementation and robust verification of the SR method. Rapid algorithms allow for automated assignment of lag time, rather than fixed assignment, and allow flux determinations while varying the length of flux averaging periods. These procedures allow for comprehensive analysis of both the physical timescales of surfaces flux, and the sensor response and uncertainty associated with the SR derived flux. Calibration of despiking criteria can be implemented quickly at low computational cost. In summary, efficient methods for computing SR flux allow implementation in novel deployments such as low-cost, continuous monitoring and on moving platforms. Future work remains to transfer efficient methods from the MATLAB development platform to open-source implementations, and to enable hardware to perform these techniques directly for realtime applications. Reducing the cost and power requirement of the required data loggers, computers, and telemetry will facilitate the extensive deployment of SR sensors to aid in describing the heterogeneity of flux across the landscape.

Data availability. All data used in this analysis and scripts implementing the algorithms described above are available online at https://doi.org/10.7267/N9X34VDS (Kelley, 2017).

Abbreviated scripts for the three example methods may be found in the Supplement. Requests for phase space despiking methods can be directed to the corresponding author.

Supplement. The supplement related to this article is available online at: https://doi.org/10.5194/amt-11-2151-2018-supplement.

Competing interests. The authors declare that they have no conflict of interest.
Acknowledgements. The authors would like to acknowledge the contributions of Bruno Luong, whose function for Cardano's solution to depressed cubic polynomials we adapted for this analysis.

Edited by: Andrew Sayer

Reviewed by: three anonymous referees

\section{References}

Allen, R., Pereira, L. S., Howell, T. A., and Jensen, M. E.: Evapotranspiration information reporting: I. Factors governing measurement accuracy, Agr. Water Manage., 98, 899-920, 2011.

Altman, Y.: Accelerating Matlab Performance, CRC Press, 2015.

Antonia, R. A. and Van Atta, C. W.: Structure functions of temperature fluctuations in turbulent shear flows, J. Fluid Mech., 84, 561-580, 1978.

Aubinet, M., Vesala, T., and Papale, D.: Eddy covariance: a practical guide to measurement and data analysis, Springer Science \& Business Media, 2012.

Baldocchi, D.: Measuring fluxes of trace gases and energy between ecosystems and the atmosphere-the state and future of the eddy covariance method, Glob. Change Biol., 20, 3600-3609, 2014.

Castellvi, F.: Combining surface renewal analysis and similarity theory: a new approach for estimating sensible heat flux, Water Resour. Res., 40, https://doi.org/10.1029/2003WR002677, 2004.

Castellví, F.: Fetch requirements using surface renewal analysis for estimating scalar surface fluxes from measurements in the inertial sublayer, Agr. Forest Meteorol., 152, 233-239, https://doi.org/10.1016/j.agrformet.2011.10.004, 2012.

Castellví, F., Perez, P. J., and Ibañez, M.: A method based on highfrequency temperature measurements to estimate the sensible heat flux avoiding the height dependence, Water Resour. Res., 38, 20-1-20-9, 2002.

Chen, W., Novak, M., Black, T. A., and Lee, X.: Coherent eddies and temperature structure functions for three contrasting surfaces. Part I: Ramp model with finite microfront time, Bound.-Lay. Meteorol., 84, 99-124, https://doi.org/10.1023/A:1000338817250, 1997a.

Chen, W., Novak, M., Black, T. A., and Lee, X.: Coherent eddies and temperature structure functions for three contrasting surfaces. Part II: Renewal model for sensible heat flux, Bound.-Lay. Meteorol., 84, 125-147, https://doi.org/10.1023/A:1000342918158, 1997b.

Edwards, A. C. and Beaver, J. M.: Investigating Cardano's Irreducible Case, Proceedings of the National Conference on Undergraduate Research (NCUR) 2015, Eastern Washington University, April 16-18 2015, available at: http://www.ncurproceedings.org/ojs/index.php/NCUR2015/ article/view/1478 (last access: 9 April 2018), 2015.

Foken, T., Leuning, R., Oncley, S. R., Mauder, M., and Aubinet, M.: Corrections and data quality control, Eddy Covariance, 85-131, Springer, 2012.

French, A. N., Alfieri, J. G., Kustas, W. P., Prueger, J. H., Hipps, L. E., Chávez, J. L., Evett, S. R., Howell, T. A., Gowda, P. H., Hunsaker, D. J., and Thorp, K. R.: Estimation of surface energy fluxes using surface renewal and flux variance techniques over 
an advective irrigated agricultural site, Adv. Water Resour., 50, 91-105, https://doi.org/10.1016/j.advwatres.2012.07.007, 2012.

Gao, W. and Li, B. L.: Wavelet analysis of coherent structures at the atmosphere-forest interface, J. Appl. Meteorol., 32, 1717-1725, 1993.

Gao, W., Shaw, R. H., and Paw U, K. T.: Observation of organized structure in turbulent flow within and above a forest canopy, Bound.-Lay. Meteorol., 47, 349-377, 1989.

Göckede, M., Rebmann, C., and Foken, T.: A combination of quality assessment tools for eddy covariance measurements with footprint modelling for the characterisation of complex sites, Agr. Forest Meteorol., 127, 175-188, 2004.

Goring, D. G. and Nikora, V. I.: Despiking Acoustic Doppler Velocimeter Data, J. Hydraul. Eng., 128, 117-126, https://doi.org/10.1061/(ASCE)0733-9429(2002)128:1(117), 2002.

Højstrup, J.: A statistical data screening procedure, Meas. Sci. Technol., 4, 153-157, https://doi.org/10.1088/0957-0233/4/2/003, 1993.

Katul, G., Hsieh, C.-I., Oren, R., Ellsworth, D., and Phillips, N.: Latent and sensible heat flux predictions from a uniform pine forest using surface renewal and flux variance methods, Bound.Lay. Meteorol., 80, 249-282, 1996.

Katul, G., Porporato, A., Cava, D., and Siqueira, M.: An analysis of intermittency, scaling, and surface renewal in atmospheric surface layer turbulence, Physica D, 215, 117-126, 2006.

Kelley, J.: Demonstration data for computational efficiency in surface renewal analysis, OSU Libraries, https://doi.org/10.7267/N9X34VDS, 2017.

Paw U, K. T., Brunet, Y., Collineau, S., Shaw, R. H., Maitani, T., Qiu, J., and Hipps, L.: On coherent structures in turbulence above and within agricultural plant canopies, Agr. Forest Meteorol., 61, 55-68, https://doi.org/10.1016/0168-1923(92)90025-Y, 1992.

Paw U, K. T., Qiu, J., Su, H.-B., Watanabe, T., and Brunet, Y.: Surface renewal analysis: a new method to obtain scalar fluxes, Agr. Forest Meteorol., 74, 119-137, https://doi.org/10.1016/01681923(94)02182-J, 1995.

Paw U, K. T., Snyder, R. L., Spano, D., and Su, H.-B.: Surface Renewal Estimates of Scalar Exchange, in Micrometeorology in Agricultural Systems, American Society of Agronomy, Crop Science Society of America, and Soil Science Society of America, Madison, WI, 455-483, https://doi.org/10.2134/agronmonogr47.c20, 2005.

Press, W. H. (Ed.): Numerical recipes: the art of scientific computing, 3rd Edn., Cambridge University Press, Cambridge, UK, New York, 2007.

Rebmann, C., Kolle, O., Heinesch, B., Queck, R., Ibrom, A., and Aubinet, M.: Data acquisition and flux calculations, Eddy Covariance, 59-83, Springer, 2012.

Shapland, T. M., McElrone, A. J., Snyder, R. L., and Paw U, K. T.: Structure Function Analysis of Two-Scale Scalar Ramps. Part I: Theory and Modelling, Bound.-Lay. Meteorol., 145, 5-25, https://doi.org/10.1007/s10546-012-9742-5, 2012a.
Shapland, T. M., McElrone, A. J., Snyder, R. L., and Paw U, K. T.: Structure Function Analysis of Two-Scale Scalar Ramps. Part II: Ramp Characteristics and Surface Renewal Flux Estimation, Bound.-Lay. Meteorol., 145, 27-44, https://doi.org/10.1007/s10546-012-9740-7, $2012 \mathrm{~b}$.

Shapland, T. M., Snyder, R. L., Paw U, K. T., and McElrone, A. J.: Thermocouple frequency response compensation leads to convergence of the surface renewal alpha calibration, Agr. Forest Meteorol., 189-190, 36-47, https://doi.org/10.1016/j.agrformet.2014.01.008, 2014.

Shaw, R. H. and Gao, W.: Detection of temperature ramps and flow structures at a deciduous forest site, Agr. Forest Meteorol., 47, 123-138, 1989.

Snyder, R. L., Spano, D., and Pawu, K. T.: Surface renewal analysis for sensible and latent heat flux density, Bound.-Lay. Meteorol., 77, 249-266, 1996.

Snyder, R. L., Spano, D., Duce, P., Paw U, K. T., and Rivera, M.: Surface renewal estimation of pasture evapotranspiration, J. Irrig. Drain. E., 134, 716-721, 2008.

Spano, D., Snyder, R. L., Duce, P., and Paw U, K. T.: Surface renewal analysis for sensible heat flux density using structure functions, Agr. Forest Meteorol., 86, 259-271, 1997.

Spano, D., Snyder, R. L., and Duce, P.: Estimating sensible and latent heat flux densities from grapevine canopies using surface renewal, Agr. Forest Meteorol., 104, 171-183, 2000.

Starkenburg, D., Metzger, S., Fochesatto, G. J., Alfieri, J. G., Gens, R., Prakash, A., and Cristóbal, J.: Assessment of Despiking Methods for Turbulence Data in Micrometeorology, J. Atmos. Ocean. Tech., 33, 2001-2013, https://doi.org/10.1175/JTECHD-15-0154.1, 2016.

Stull, R. B.: An introduction to boundary layer meteorology, Springer, 1988.

Suvočarev, K., Shapland, T. M., Snyder, R. L., and Martínez-Cob, A.: Surface renewal performance to independently estimate sensible and latent heat fluxes in heterogeneous crop surfaces, J. Hydrol., 509, 83-93, https://doi.org/10.1016/j.jhydrol.2013.11.025, 2014.

The Mathworks Inc.: Matlab R2016b, The MathWorks Inc., Natick, MA, 2016.

Van Atta, C. W.: Effect of coherent structures on structure functions of temperature in the atmospheric boundary layer, Arch. Mech., 29, 161-171, 1977.

Van Atta, C. W. and Park, J.: Statistical self-similarity and inertial subrange turbulence, in: Statistical Models and Turbulence, 402426, Springer, 1972.

Vickers, D. and Mahrt, L.: Quality control and flux sampling problems for tower and aircraft data, J. Atmos. Ocean. Tech., 14, 512 526, 1997. 